\title{
Assessment of the effects of antidepressants on contractile function of guinea pig urinary bladder and urethra
}

\author{
Keisuke Obara ${ }^{1}$, Hiroko Suzuki ${ }^{1}$, Satomi Miyatani ${ }^{1}$, Satoko Imanaka ${ }^{1}$, Hiroka Fukuhara ${ }^{1}$, \\ Fumiko Yamaki ${ }^{1}$, Takashi Yoshio ${ }^{2}$, Yoshio Tanaka ${ }^{1}$ \\ ${ }^{I}$ Department of Chemical Pharmacology, Faculty of Pharmaceutical Sciences, Toho University, Japan, ${ }^{2}$ Department of \\ Clinical Pharmacology, Faculty of Pharmaceutical Sciences, Toho University, Japan
}

Background: We aimed to find antidepressants that possessed a minimal incidence of drug-induced urinary disorders by investigating 18 clinically available antidepressants in Japan for potential inhibitory/augmentative effects on acetylcholine/adenosine triphosphate (ACh/ATP)-induced urinary bladder (UB) contractions and noradrenaline (NA)induced urethral contractions.

Methods: We studied the effects of 18 antidepressants on the concentration-response curves for ACh/ATP-induced contractions in guinea pig (GP) UB strips and also for NA-induced contractions in GP urethra strips. When inhibitory or augmentative effects were significant, the experimental concentrations were compared with clinically achievable blood concentrations to check for clinical significance.

Results: (1) Tricyclic antidepressants (imipramine, amitriptyline, trimipramine, clomipramine, nortriptyline, and amoxapine), maprotiline (a tetracyclic antidepressant), and mirtazapine (a noradrenergic and specific serotonergic antidepressant; NaSSA) competitively inhibited ACh-induced UB contractions at clinically achievable concentration ranges. Mianserin (a tetracyclic antidepressant), paroxetine and sertraline (selective serotonin reuptake inhibitors; SSRIs), and duloxetine (a serotonin norepinephrine reuptake inhibitor; SNRI) also competitively inhibited ACh-induced contraction, albeit at concentrations that substantially exceeded clinically achievable blood levels. In contrast, fluvoxamine and escitalopram (SSRIs), milnacipran (an SNRI), trazodone (a serotonin 2A receptor antagonist), sulpiride (a dopamine $\mathrm{D}_{2}$ receptor antagonist), and aripiprazole (a dopamine partial agonist) did not significantly affect ACh-induced contraction at concentrations up to $10^{-5} \mathrm{M}$. (2) None of the antidepressants affected ATP-induced UB contractions within clinically achievable concentration ranges. (3) Nortriptyline, amoxapine, maprotiline, paroxetine, milnacipran, and duloxetine significantly increased NA-induced contraction at $10^{-6} \mathrm{M}$ in the distal portion of the urethra. In the proximal portion of urethra, imipramine, clomipramine, trimipramine, amitriptyline, and nortriptyline significantly suppressed NAinduced contraction at $10^{-6} \mathrm{M}$. Fluvoxamine, sertraline, escitalopram, mirtazapine, and sulpiride did not significantly affect NA-induced contractions in either portion of the urethra.

Conclusions: These findings suggest that among the tested antidepressants, two SSRIs (fluvoxamine and escitalopram) and sulpiride have the least influence on contractile functions of the UB and urethra. Thus, these antidepressants are unlikely to lead to urinary disorders that are caused by suppression/augmentation of ACh-, ATP-, and NA-induced contractions. 